\title{
Different Risk Measures: Different Portfolio Compositions?
}

\author{
A Paper Presented at \\ The $11^{\text {th }}$ Annual European Real Estate Society (ERES) Meeting \\ Milan, Italy, June 2004
}

\author{
Peter Byrne and Stephen Lee \\ Centre for Real Estate Research \\ The University of Reading Business School \\ Reading, RG6 6AW, UK
}

Phone: +44 (0)118 3786338 E-mail: S.L.Lee@reading.ac.uk

Phone: +44 (0)118 3786340 E-mail: P.J.Byrne@reading.ac.uk

\begin{abstract}
Traditionally, the measure of risk used in portfolio optimisation models is the variance. However, alternative measures of risk have many theoretical and practical advantages and it is peculiar therefore that they are not used more frequently. This may be because of the difficulty in deciding which measure of risk is best and any attempt to compare different risk measures may be a futile exercise until a common risk measure can be identified. To overcome this, another approach is considered, comparing the portfolio holdings produced by different risk measures, rather than the risk return trade-off. In this way we can see whether the risk measures used produce asset allocations that are essentially the same or very different.

The results indicate that the portfolio compositions produced by different risk measures vary quite markedly from measure to measure. These findings have a practical consequence for the investor or fund manager because they suggest that the choice of model depends very much on the individual's attitude to risk rather than any theoretical and/or practical advantages of one model over another.
\end{abstract}

Keywords: Alternative Risk Models, Portfolio Compositions, Similarity Indices 


\section{Different Risk Measures: Different Portfolio Compositions?}

\section{Introduction}

Selecting the appropriate portfolio of assets in which to invest is an essential component of real estate fund management. Although a large proportion of portfolio selection decisions are still taken on a qualitative basis, quantitative approaches to selection are increasingly being employed. Markowitz (1952) established a quantitative framework for asset selection into a portfolio that is now well known. In this it is assumed that asset returns follow a multivariate normal distribution or that investors have a quadratic utility function. This approach shows that characteristics of a portfolio of assets can be completely described by the mean and the variance (risk) and so is described as the mean-variance (MV) portfolio model. For a particular universe of assets, the set of portfolios of assets that offer the minimum risk for a given level of return form the efficient frontier. The portfolios on the efficient frontier can be found by quadratic programming and such problems can now be solved easily in spreadsheet programs (see Byrne and Lee, 1994a, 1994b). The solutions are optimal and the selection process can be constrained by practical considerations, such upper and lower bounds, which can be written as linear constraints. The weakness of the MV approach however is that the underlying assumptions of multivariate normality or that investors have a quadratic utility function are not sustainable. This has led researchers to develop portfolio asset allocation models using other measures of risk that have many theoretical and practical advantages over MV. Even so, the MV approach remains the most popular approach to the asset allocation problem.

This may be because deciding which measure of risk is 'best' is still unresolved (Stone, 1973). Cheng and Wolverton (2001) for example, highlight the difficulty of comparing portfolios based on different risk criteria. They find that each approach produces results that minimise risk, but only in its own space. When the portfolio compositions of one risk measure are used to calculate the risk and return trade-off in another risk space the results are always "inferior" to the solutions produced inside that risk space. They argue that any attempt to find the portfolio model that offers the best risk return trade-off is likely to be futile until a common risk measure can be identified.

In this paper, another approach, which overcomes this, is considered (Phillips, 1993). The portfolio asset holdings and weights produced by different risk measures are compared, rather than the conventional risk/return trade-off. In this way it is possible to see whether different risk measures produce asset allocations that are essentially the same or radically different. So, for instance, if the portfolio compositions produced by different risk models, i.e. the assets chosen and the weights assigned to the them, are essentially the same then there is little to be gained from using one risk measure or another. In contrast, if the portfolio compositions produced by the different risk models are substantially different, the choice of risk measure becomes crucial to the investor.

The paper is structured as follows. The next section discusses the various risk measures used in the study. Section 3 presents the data. The following section provides a brief discussion of the various optimisation models used and shows the average results of the various optimisations. A number of similarity indices are then calculated and discussed in section 5 . Section 6 concludes the paper. 


\section{Risk Measures}

The variance remains the most commonly used risk measure in portfolio optimisation models. Markowitz (1952) showed that if risk is measured by the variance of returns and expected return by the mean of returns, then uncertain investments can be ordered by their ranking in MV space. The variance is defined as:

$$
\operatorname{Var}=\frac{1}{T} \sum_{t=1}^{T}\left(R_{t}-\bar{R}\right)^{2}
$$

Although the MV model is the most popular approach, it relies on the assumptions that returns are either normally distributed or that the investor's utility function is quadratic. If either of these conditions hold, it can be shown that choosing among risky investments is compatible with the maximisation of an investor's expected utility (Tobin, 1958). Many authors have pointed out however that both of the assumptions underlying the MV model generally do not hold - either theoretically or in practice. Apart from the criticisms of the assumptions underpinning the MV model, it has been argued also that the use of the variance as a measure of risk implies that investors are indifferent between returns above and below the mean. Clearly however, most risk-averse investors are generally more concerned with risk below some target level of return, be it the mean or some other benchmark.

In order to overcome the difficulties associated with the MV model, Markowitz (1952) and others proposed the semi-variance. The SV concentrates on the returns below the mean (expected return) so that the SV is defined as

$$
\begin{aligned}
& S V=\frac{1}{T} \sum_{t=1}^{T}\left(\min \left[0, R_{t}-E(R)\right]\right)^{2} \\
& \text { subject to } R_{t}<E(R)
\end{aligned}
$$

Bawa (1975) and Bawa and Lindenberg (1977) generalised this idea by suggesting models based on Lower Partial Moments (LPM) over $n$ orders (see for example, Sing and Ong, 2000). LPMs of order 2 are measures of portfolio risk that focus on returns below some target level, so that for example, the semi-variance is just a special case LPM when $R_{\tau}$ equals the $E(R)$.

The LPM $(n=2)$ is thus :

$$
\begin{aligned}
& L P M=\frac{1}{T} \sum_{t=1}^{T}\left(\min \left[0, R_{t}-R_{\tau}\right]\right)^{2} \\
& \text { again, subject to } R_{t}<R_{\tau}
\end{aligned}
$$

In the investment literature, the target $R_{\tau}$ is usually the minimum return that the investor would be willing to receive. The target is often set to the risk-free rate or it could be zero: that is negative returns are to be avoided ${ }^{1}$. Alternatively, the target could be set to the return of the benchmark index (B) that the manager is expected to outperform. In that case a new variable (R-B) can be defined and the target set to zero. A target of zero can be considered as the general case and is the one used in this paper (LPMZ).

\footnotetext{
1 The risk-free rate is not used as the target return in the optimisations below because this would mean
} that the target rate would be a non-constant value when a constant value is assumed. 
The MV and SV models require the use of complex non-linear numerical algorithms to solve the portfolio problem. The practical application of such models was severely limited until computers were powerful enough to handle even the smallest problems. Sharpe (1971) commented that if the portfolio problem could be formulated as a linear programming problem, the prospect for practical application would be greatly enhanced. Young (1998) has proposed such a solution based on the "minimax" (MM) rule. The MM rule has a long tradition in models of uncertainty because, in situations with conflicting alternatives, the most rational choice is that which seeks to minimise the maximum loss (negative gain). Given an historic time series of returns, the optimum portfolio under the MM rule is defined as that which would minimise the maximum loss over all past periods, subject to a restriction that some minimum average return is achieved across the observed time periods. Hence the LP can be defined as follows:

$$
\max M_{P}
$$

Subject to

$$
\begin{aligned}
& \sum_{i=1}^{N} w_{i} r_{i t}-M_{P} \geq 0, t=1, \quad, T, \\
& \sum_{i=1}^{N} w_{i} \bar{r}_{i} \geq B,
\end{aligned}
$$

and as usual

$$
\sum_{i=1}^{N} w_{i}=1
$$

and

$$
w_{i} \geq 0, i=1, \ldots, N \text {. }
$$

The MM portfolio model has a number of advantages over the MV model. Young shows that the MM rule corresponds approximately to an expected utility function that is more extreme than that implied by the MV rule, having a strong absolute aversion to downside risk. The MM approach is akin to the LPM rule, especially if the level of maximum loss that the investor requires is set to zero or the risk free rate. MM linear programming solutions can also accommodate complex decision variables such as integer values, e.g. fixed transaction costs, which are difficult to incorporate into the quadratic programming model used by the MV rule. In addition, the MM rule has logical advantages over the MV rule if returns are non-normally distributed. Based on the results of simulation studies, Young concludes that the MM rule provides a convenient and useful approach to portfolio selection.

All these measures of risk are sensitive to outliers in the data because the mean differences are squared. Squaring gives such outliers a disproportionate influence in the calculation of the measures of risk. In a similar way, LPM measures are sensitive to observations that are distant from their target. The MM rule also will be affected strongly by such outliers, because it specifically minimises the maximum negative return. A measure of variability that is less sensitive to outliers is the mean absolute deviation (MAD). This prompted the development of portfolio optimisations that use the MAD as a measure risk (Konno, 1989). Such models have a number of 
advantages. First, Konno and Yamazaki (1991) show that the MAD approach is equivalent to the MV model if the returns are multivariate normally distributed. Secondly, the MAD model produces optimal portfolios without the need to calculate the covariance matrix and so can be used in situations when $N$, the number of assets, is greater than $T$, the number of time periods over which the analysis is performed. Finally, Konno and Shirakawa (1994) show that the MAD model can handle large problems in real time. However a limitation of this approach is that the computational savings from the use of MAD objective functions may in some cases be outweighed by the loss of information from the (unused) covariance matrix (Simaan, 1997).

The MAD is defined by:

$$
M A D=\frac{1}{T} \sum_{t=1}^{T}\left|R_{t}-E(R)\right|
$$

Other portfolio models using different measures of risk have been and continue to be developed. MV, SV, LPMZ, MM and MAD models are considered in this study to keep the comparisons to a reasonable number.

\section{Data}

The data used in this study are the total monthly returns for the 10 market segment indices used by the Investment Property Databank (IPD) in their standard performance analysis reports to investors. The IPD monthly indices are based on the individual property data from 55 institutional investors and cover more than 2,500 properties valued at $£ 2.7$ billion at the end of 2002. Details of the construction methods are available in IPD (2002). Tests performed by IPD have suggested that this 10 segment categorisation maximises the explanatory variance in returns across individual properties and is the most effective split for asset allocation optimisation (Frodsham and Key, 1996).

The 10 market segments are: Standard Retail Southeast (SRSE); Standard Retail Rest of UK (SRRUK); Shopping Centres (SHC); Retail Warehouses (RW); Offices in the City of London (OCITY); Offices in the West End (OWE); Offices Rest of Southeast (ORSE); Offices Rest of UK (ORUK); Industrials Southern and Eastern (ISE) and Industrials Rest of UK (IRUK). The data cover the period January 1987 to December 2002, a total of 192 monthly returns. Summary statistics for the data are presented in Table 1. 
Table 1: Statistics of the IPD 10 segment categorisation: Monthly Data 1987:1 - 2002:12

\begin{tabular}{l|cccccccccc}
\hline & SRSE & SRRUK & SHC & RW & OCITY & OWE & ORSE & ORUK & ISE & IRUK \\
\hline Mean & 0.72 & 0.71 & 0.81 & 1.12 & 0.49 & 0.84 & 0.77 & 0.92 & 1.01 & 1.16 \\
SD & 0.8 & 0.72 & 0.86 & 1 & 1.55 & 1.43 & 0.99 & 1.03 & 0.95 & 0.99 \\
Skewness & 0.93 & 1.26 & -0.68 & 0.08 & -0.97 & 0.38 & 0.53 & 1.28 & 0.7 & 1.72 \\
Kurtosis & 4.88 & 8.11 & 6.86 & 6.57 & 9.37 & 5.44 & 4.33 & 5.52 & 4.31 & 6.87 \\
JB Test & 56 & 259.2 & 134.1 & 102.4 & 355.3 & 52.3 & 23.2 & 103 & 29.36 & 214.5 \\
\hline
\end{tabular}

Table 1 shows that the segment with the highest average return overall was Industrials in the Rest of the UK (IRUK), while that the segment with the lowest risk was Standard Retail Rest of the UK (SRRUK). However, the segment with the highest overall risk, Offices in the City of London (OCITY), also had the lowest average returns. In general the market segments display positive skewness, with IRUK showing the highest value, but two market segments SHC and OCITY do display negative skewness, OCITY showing significant negative skewness. All the assets display significant positive kurtosis, (i.e. they are leptokurtic) that is more peaked than the normal distribution. This is a well known feature of real estate data. Lizieri and Ward (2001) argue that this comes from the presence of a high proportion of zero returns and too few larger negative and positive returns, which can be attributed to the thinly traded nature of direct property, where new information is infrequent and is only slowly impounded into valuations. Consequently, Jarque-Bera tests show that the data were not normally distributed at the usual levels of significance.

\section{Asset Allocation Comparisons}

In order to investigate the potential differences between the portfolio allocations produced by the alternative risk measures, a simple asset allocation problem is examined using the data discussed in the previous section. Specifically, this is for an investor whose portfolio is updated on a quarterly basis using the previous 36 month's data (hence there are 53 points at which rebalancing occurs). Rather than considering the whole efficient frontier however, as Cheng (2001) does, the minimum risk (MR) portfolio is examined here since this optimisation involves only the measure of risk. The results for the 53 optimisations are shown in Table 2. Table 2 contains the average portfolio holdings in each 'asset' and the comparative risk of the optimisations in MV space.

Table 2: Average Portfolio Holdings and Risk/Return Trade-off: 53 Optimisations MR Portfolio

\begin{tabular}{l|ccccccccccc|c}
\hline & \multicolumn{7}{|c|}{ Average Percentage Holding in Market Segment } & MV \\
\hline & SRSE & SRRUK & SHC & RW & OCITY & OWE & ORSE & ORUK & ISE & IRUK & Risk \\
\hline MV & 12.1 & 20.8 & 14.4 & 0.5 & 9.2 & 7.1 & 3.1 & 22.2 & 3.3 & 7.4 & 0.48 \\
MAD & 10.8 & 19.8 & 15.5 & 0.2 & 9.7 & 7.6 & 3.3 & 24.6 & 2.7 & 5.8 & 0.50 \\
MM & - & 0.4 & 2.1 & 19.3 & 3.1 & 12.8 & 0.3 & 12.4 & 27.4 & 22.2 & 0.65 \\
SV & 21.9 & 13.8 & 4.8 & 0.2 & 45.5 & 1.4 & 2.6 & 8 & 1.1 & 0.7 & 0.58 \\
LPMZ & 9.3 & 3 & 3.6 & 28.3 & - & 3.9 & 3.8 & 6.8 & 4 & 37.3 & 0.74 \\
\hline
\end{tabular}


Figure 1 and Table 2 show that the mean allocations produced by the alternative risk models are significantly different to the MV approach, except for those in the MAD model. This is to be expected because the data display considerable non-normality. In particular, the LPMZ shows a dramatic shift in allocation away from the market segment with significant negative skewness (OCITY) to the segment with significant positive skewness (IRUK). The MM model also shows a similar aversion to negatively skewed segments, with $63 \%$ of the average portfolio allocation in ORUK, ISE and IRUK. This highlights the link between downside risk and negative skewness.

The portfolio weights produced by the alternative risk models were then used to calculate the average risks and returns in MV space. The results in Table 2 show a number of features of interest. First, all the alternative risk measures produce risks that are "inferior" to those produced by MV analysis. This supports the conclusions of Cheng and Wolverton (2001) that the risk measures, although optimal in their own risk space, always produce results that are inferior in another risk space and the question of which risk measure is the "best" cannot be addressed adequately by such comparisons. Second, the results from the MAD and SV models are the closest to those of the MV solution. This agrees with Byrne and Lee (1999) who show that that the MAD approach produces portfolio weights and has risk/return tradeoffs that are similar to the MV model. 


\section{Asset Allocation Similarity}

To see whether similar portfolio allocations occur when different risk measures are used, two issues need to be addressed (Philips, 1993). First, to what extent do the same assets appear in each optimisation model? Second, for those assets that are contained in each solution, to what extent do they appear in similar proportions? In order to assess this, the number of assets and their weights in each optimal portfolio were identified. From these it was possible to find those assets that are common, (overlap), in each model and also the extent to which the weights are similar between the different risk measures. The average results are presented in Table 3.

Panel A of Table 3 shows that on average the $53 \mathrm{MV}$ solutions contained 4.1 assets, with a maximum of 7 and a minimum of 1 . By comparison, the MAD solution was made up, on average, of 3.3 assets, with a maximum of 6 and a minimum of 1 . Panel A shows that all the models produce average solutions which, based on a onetailed t-test, are significantly less than the MV solutions.

Table 3: Average Portfolio Overlap, Weight and Similarity Indices: Compared with the Minimum Risk MV Solution

\begin{tabular}{|c|c|c|c|c|c|}
\hline $\begin{array}{l}\text { Panel A: Average } \\
\text { Holdings }\end{array}$ & MV & MAD & MM & sv & LPMZ \\
\hline Average & 4.1 & 3.3 & 3.1 & 3.0 & 3.2 \\
\hline Max & 7 & 6 & 5 & 6 & 8 \\
\hline Min & 1 & 1 & 1 & 1 & 1 \\
\hline $\begin{array}{l}\text { Panel B: Holdings in } \\
\text { Common }\end{array}$ & MV & MAD & MM & SV & LPMZ \\
\hline MV & 4.1 & & & & \\
\hline MAD & 3.1 & 3.3 & & & \\
\hline MM & 1.4 & 1.1 & 3.1 & & \\
\hline SV & 2.5 & 2.2 & 1.0 & 3.0 & \\
\hline LPMZ & 1.6 & 1.1 & 1.4 & 0.9 & 3.3 \\
\hline Panel C: Overlap Indices & MV & MAD & MM & SV & LPMZ \\
\hline MV & 100.0 & & & & \\
\hline MAD & 74.3 & 100.0 & & & \\
\hline MM & 25.6 & 22.2 & 100.0 & & \\
\hline SV & 56.4 & 56.2 & 19.3 & 100.0 & \\
\hline LPMZ & 20.8 & 14.5 & 31.2 & 12.3 & 100.0 \\
\hline Panel D: Weight Indices & MV & MAD & MM & SV & LPMZ \\
\hline MV & 100.0 & & & & \\
\hline MAD & 80.2 & 100.0 & & & \\
\hline MM & 16.6 & 15.1 & 100.0 & & \\
\hline SV & 48.9 & 46.6 & 8.2 & 100.0 & \\
\hline LPMZ & 15.3 & 11.2 & 45.1 & 6.9 & 100.0 \\
\hline Panel E: Similarity Indices & MV & MAD & MM & SV & LPMZ \\
\hline MV & 100.0 & & & & \\
\hline MAD & 60.5 & 100.0 & & & \\
\hline MM & 7.4 & 5.2 & 100.0 & & \\
\hline SV & 31.7 & 29.9 & 3.0 & 100.0 & \\
\hline LPMZ & 7.0 & 4.1 & 15.3 & 1.8 & 100.0 \\
\hline
\end{tabular}

Panel B of Table 3 shows that the number of assets in the minimum risk portfolios that overlap, or are common to both the MV and MAD optimisations, is 3.1. This means that on average, 3.1 of the 4.1 assets that make up the MV portfolios are also part of the 3.3 assets that form the MAD portfolios. Similar calculations are made for the other risk measures. Panel B shows that the risk measure with the least assets 
in common with the others, is the LPMZ. Using these raw numbers portfolio overlap, weight and similarity indices were then calculated (see Philips, 1993 for details).

As an example, the average portfolio overlap index for the MAD against the MV is defined as the ratio of the number of assets that overlap between the two risk measures to the average number of assets, in the union between the two risk measures. The union between the two risk measures is equal to the average number of assets in the MV solution (4.1) plus the number of assets in the MAD solution (3.3), minus the number of assets in common (3.1), which in this case is equal to $(4.1+3.3-3.1)=4.3$. Thus while on average the MV and MAD solutions contained (4.1) and (3.3) assets, only 3.1 were in common to both results, leaving 4.3 assets that appear in only one solution. The ratio of the overlap between the two risk measures to the union between the two risk measures is therefore $3.3 / 4.3=72.4 \%$. Thus, on average, $72.4 \%$ of holdings contained in the MV solution are also contained in the MAD solutions. Other portfolio overlap indices were calculated in the same way. The results are given in Panel $\mathrm{C}$ of Table 3.

The calculation of portfolio overlap indices only addresses one facet of the similarity or dissimilarity of portfolio compositions. When two portfolios contain exactly the same assets the portfolio overlap index will be $100 \%$. However, the weights within such portfolios could vary markedly. This has important investment implications.

To test the similarity between the weights attached to assets held in common by two portfolios a portfolio weight index can be constructed. The index is measured by summing the minimum weight attached to each asset that overlaps two portfolio solutions. For example, column 2 of Table 3 shows a portfolio overlap index of $74.3 \%$ between the MV and MAD models. On the other hand, the sum of the minimum weights found in the MR portfolios between the two risk measures for the holdings that are common in both solutions is $80.2 \%$. In other words, $80.2 \%$ of the holdings in the MR portfolio of the MV solution are common to the MAD solution. The portfolio weight indices for the alternative risk measures are calculated in the same way. The results are presented in Panel D of Table 3.

Multiplying the portfolio overlap indices by the portfolio weight indices gives the proportion of assets in common to both risk measures with similar weights, i.e. a portfolio similarity index. These are shown in Panel E of Table 3. In the case of the MR portfolios for the MV and MAD solutions, $60.5 \%$ of the holdings in the MV optimisation are also in the MAD optimisation with similar portfolio weights. The average portfolio similarity indices of the risk measures, compared with the alternatives, are calculated in the same way.

Table 3 shows a number of features of interest. First, given the results in Table 2, by comparison with the MV solutions, the MAD model shows the largest similarity with a value of $60.5 \%$, while LPMZ shows the least similarity; $7 \%$. Second, in comparison with the other models, LPMZ with MM show the largest similarity (15.3\%), but LPMZ with SV the least (1.8\%). In addition, Figure 2 shows that the average values (Table 3: Panel E) hide a great deal of variability over the 53 optimisations. Nonetheless, the MAD values are closest to the MV solutions; closely followed by the SV results, with the MM and LPMZ models showing similarity values that are substantially lower than the MV optimisations. 


\section{Conclusions}

The measure of risk used traditionally in portfolio optimisation models is the variance. This is in spite of the fact that other measures of risk have many theoretical and practical advantages. Given the advantages that these other measures of risk present, it seems puzzling that they are not used more frequently. However, if it can be shown that these alternative approaches produce asset allocations that are essentially the same as those produced by the MV model then there would be little to be gained from its continuing use. In order to investigate this issue this study tested the proposition that different measures of risk produce minimum risk portfolios that are essentially the same in terms of asset allocations, using monthly data over the period January 1987 to December 2002. The results indicate that the portfolio compositions produced by different risk measures vary markedly from risk measure to risk measure. Of the various alternatives examined the one that comes closest to the MV model is the MAD. The MAD approach produced asset allocations that are most similar to those produced by MV optimisation and a risk level that is very similar to that of the MV model in MV space. In contrast, the LPMZ and MM models produce asset allocations that are least like those of the MV model, and risk values in MV space that are substantially higher. These findings have a practical consequence for the investor because they suggest that the choice of model depends very much on individual attitudes to risk rather than any theoretical and practical advantages of one model over another. 


\section{References}

Bawa, V.S. (1975) Optimal Rules for Ordering Uncertain Prospects, Journal of Financial Economics, 2, 1, 95-121.

Bawa, V.S. and Lindenberg, E.B. (1977) Capital Market Equilibrium in a Mean-Lower Partial Moment Framework, Journal of Financial Economics, 5, 2, 189-200.

Byrne, P.J. and Lee, S.L. (1994a) Computing Markowitz Efficient Frontiers Using a Spreadsheet Optimiser, Journal of Property Finance, 5, 1, 1994, 58-66.

Byrne, P.J. and Lee, S.L. (1994b) Real Estate Portfolio Analysis Using a Spreadsheet Optimiser. Journal of Property Finance, 5, 4, 1994, 19-31.

Byrne, P. and Lee, S.L. (1999) The Place of Property in an Australian Multi-Asset Portfolio: A Comparison of the MPT and MAD Optimisation Methods, Australian Land Economics Review, 5, 1, 21-28.

Cheng, P. (2001) Comparing Downside-Risk and Mean-Variance Analysis Using Bootstrap Simulation, Journal of Real Estate Portfolio Management, 7, 3, 225-238.

Cheng, P. and Wolverton, M.L. (2001) MPT and the Downside Risk Framework: A Comment on Two Recent Studies, Journal of Real Estate Portfolio Management, 7, 2, 125-131.

Frodsham, M. and Key, T. (1996) Segmentation of the UK Property Market, Paper presented at the European Real Estate Society Conference, Belfast.

IPD (2002) UK Annual Index, Investment Property Databank Ltd, London.

Konno, H. (1989) Piecewise Linear Risk Functions and Portfolio Optimization, Journal of the Operations Research Society, Japan, 33, 139-156.

Konno, H. and Yamazaki, H. (1991) Mean-Absolute Deviation Portfolio Optimization Model and its Applications to the Tokyo Stock Market. Management Science, 37, 5, 519-531.

Konno, H. and Shirakawa, H. (1994) Equilibrium Relations in a Capital Asset Market: A Mean-Absolute Deviation Approach, Financial Engineering and the Japanese Markets, 1, 21-35.

Lizieri, C. and Ward, C.W.R. (2000) The Distribution of Real Estate Returns. In: Knight, J. and Satchell, S. (eds) Return Distributions in Finance. London: Butterworth-Heinemann, 2000, 47-74.

Markowitz, H. (1952) Portfolio Selection, Journal of Finance, 7, 1, 77-91.

Phillips, H.E. (1993) Portfolio Optimization Algorithms, Simplified Criteria, and Security Selection: A Contrast and Evaluation, Review of Quantitative Finance and Accounting, 3, 91-97.

Sing, T.F. and Ong, S.E. (2000) Asset Allocation in a Downside Risk Framework. Journal of Real Estate Portfolio Management, 6, 3, 213-223. 
Sharpe, W. (1971) Linear Programming Formulation of the General Portfolio Selection Problem, Journal of Financial and Quantitative Analysis, 8, 621-636.

Simaan, Y. (1997) Estimation Risk in Portfolio Selection: the Mean Variance Model Versus the Mean Absolute Deviation Model, Management Science, 43, 437-446.

Stone, B. (1973) A General Class of Three-Parameter Risk Measures, The Journal of Finance, 28, 675-685.

Tobin, J. (1958) Liquidity Preference as Behaviour towards Risk, Review of Economic Studies, 25, 65-86.

Young, M.R. (1998) A Minimax Portfolio Selection Rule with Linear Programming Solution, Management Science, 44, 5, 673-683. 
Figure 1: Asset Composition of Minimum Risk Portfolios

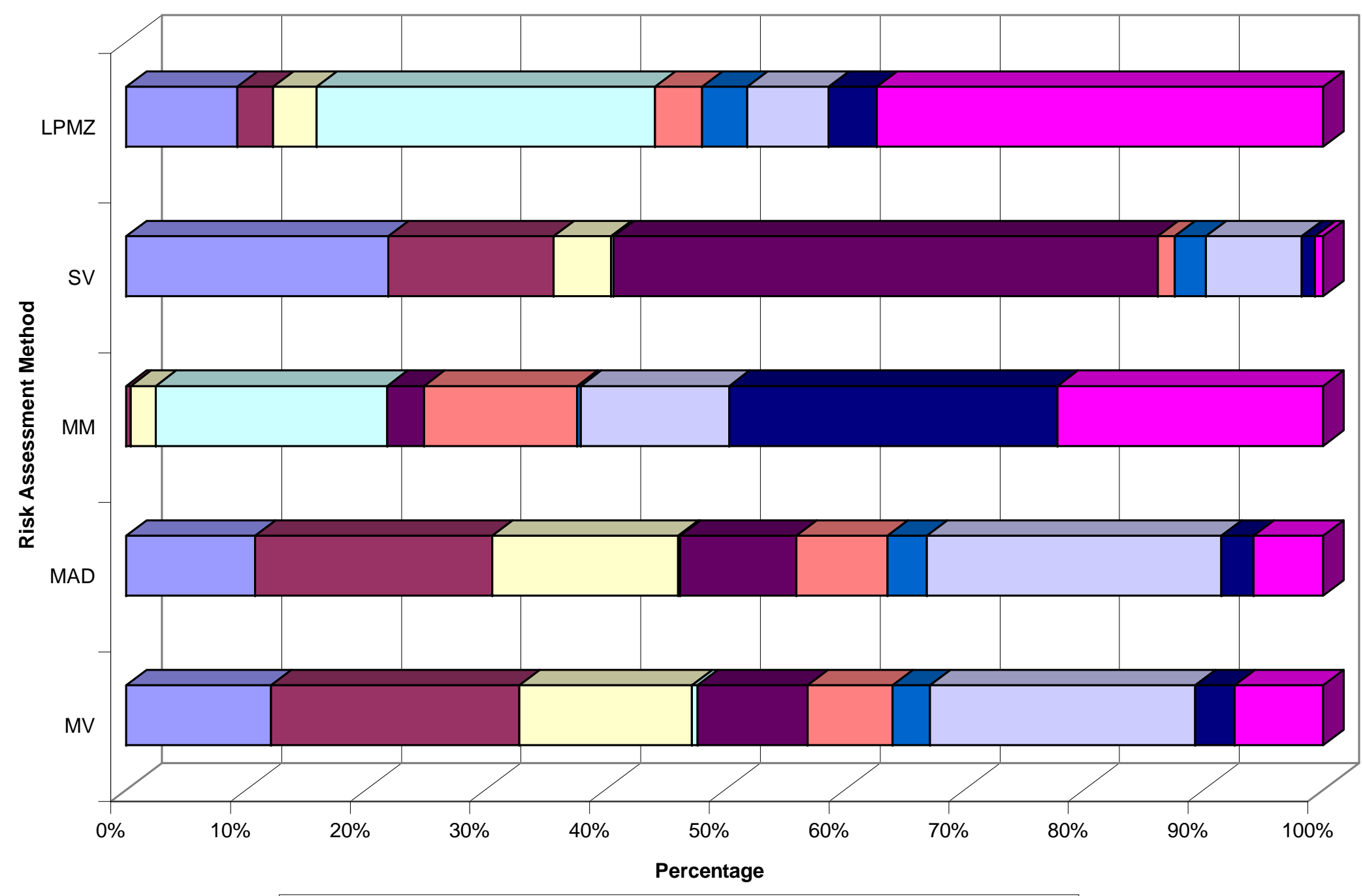

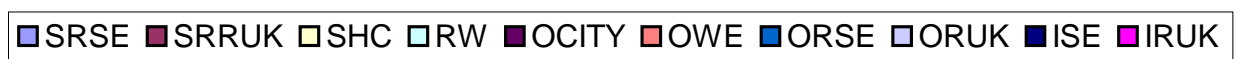


Figure 2: Similarity Indices Relative to MV Portfolio Compositions

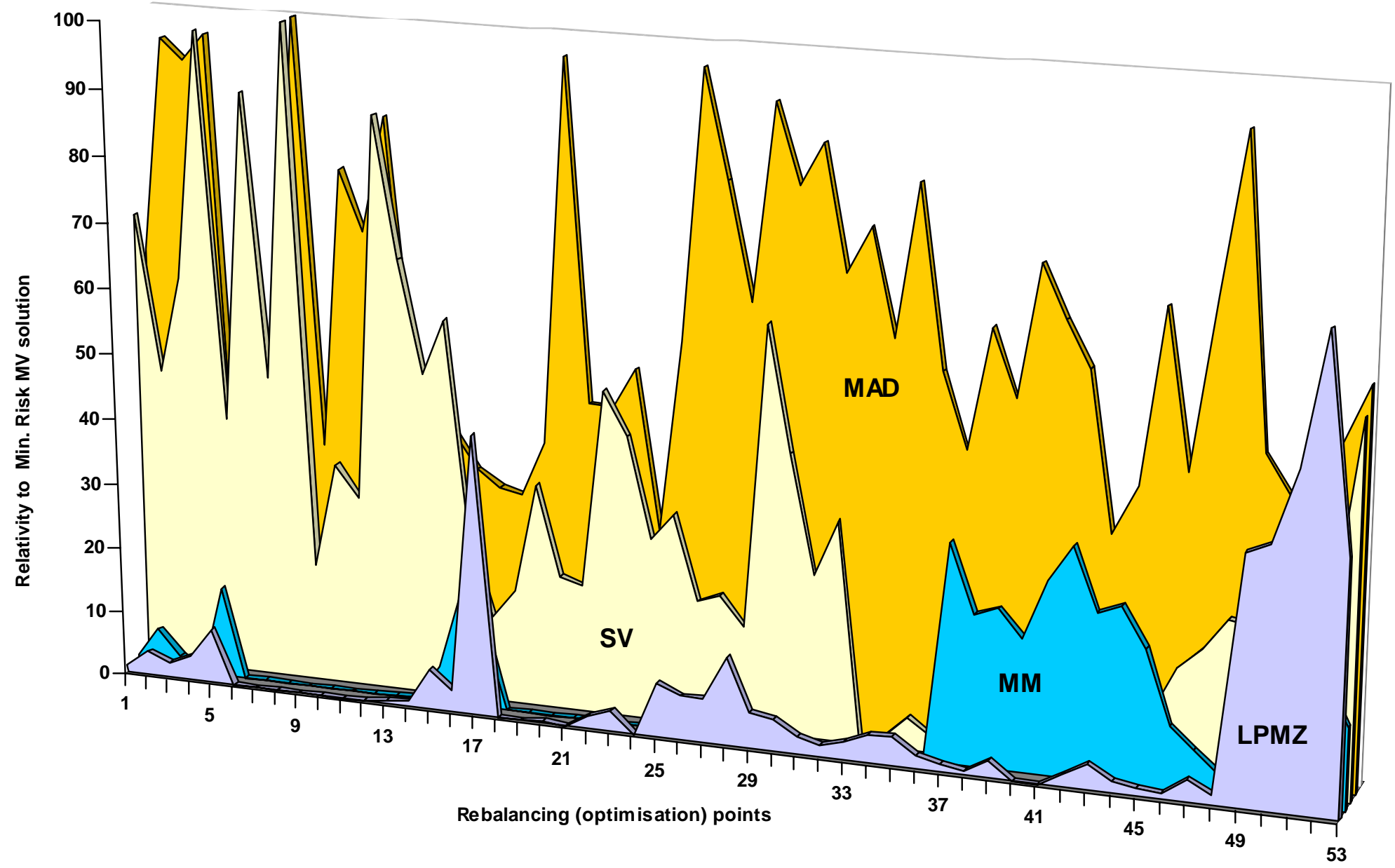

